\title{
Plasma Concentrations of Carbohydrates and Sugar Alcohols in Term Newborns after Milk Feeding
}

\author{
LAURA D. BROWN, CLAUDIO CAVALLI, JERI E. F. HARWOOD, ANNACHIARA CASADEI, CECILIA C. TENG, \\ CRISTINA TRAGGIAI, GIOVANNI SERRA, GIULIO BEVILACQUA, AND FREDERICK C. BATTAGLIA
}

\begin{abstract}
Department of Pediatrics [L.D.B., J.E.F.H., C.C.T., F.C.B.], University of Colorado Denver, Aurora, Colorado 80045; Department of Neonatology [C.C., A.C., G.B.], University of Parma, Parma 43100, Italy; Department of Neonatology [C.T., G.S.], Institute G. Gaslini,
\end{abstract} Genoa 16147, Italy

\begin{abstract}
Nonglucose carbohydrates such as galactose, mannose, and inositol play a clinically important role in fetal and neonatal nutrition, though little is known about their metabolism in the neonate. The aim of this study was to determine whether postprandial changes in plasma carbohydrate and sugar alcohol concentrations are affected by clinical variables such as postnatal age (PNA), milk type, feeding volume, or feeding duration in term newborns. Neonates $(n=26)$ taking intermittent enteral feedings were enrolled. Blood samples were obtained at baseline (immediately before the start of a feeding) and at 2-3 subsequent time points up to $110 \mathrm{~min}$. Postprandial rise was only observed for plasma glucose concentrations [Glu] and plasma galactose concentrations [Gal] and clinical variables did not predict this change. Despite equimolar delivery in milk, the median of [Glu] rise minus [Gal] rise from baseline to second postprandial plasma sample was $674 \mu \mathrm{M}(-38,3333 \mu \mathrm{M} ; p<$ 0.0001 ), reflecting efficient hepatic first-pass metabolism of galactose. A significant PNA effect on [Gal] was observed such that for each day PNA there was an $18 \%$ decrease in [Gal] $(p=0.03)$. [Gal] are a function of PNA, suggesting maintenance of a significant ductus venosus shunt in term infants. (Pediatr Res 64: 189-193, 2008)
\end{abstract}

$\mathrm{H}$ istorically considerable attention has been focused on neonatal glucose metabolism because glucose is the major energy substrate for the neonate and because of the relatively high frequency of hypoglycemia in newborns, particularly infants of diabetic mothers (IDMs) and infants with IUGR. Several recent studies, however, have highlighted the importance of carbohydrates other than glucose in fetal and neonatal nutrition. Several sugar alcohols (polyols), such as inositol, sorbitol, and erythritol, are concentrated in embryonic fluid (1) and in both human and ovine fetal plasma $(2,3)$ compared with maternal plasma. Furthermore, inositol may be important in the pathogenesis of neural tube defects in IDMs (4) and prevention of retinopathy and chronic lung disease in preterm infants $(5,6)$. Galactose is an important carbohydrate for energy production in the first few months of life by serving as the main substrate for hepatic glycogen synthesis (7). Several free carbohydrates, oligosaccharides, and sugar alco-

Received February 7, 2008; accepted March 14, 2008.

Correspondence: Laura D. Brown, M.D., UCDHSC Perinatal Research Center, F441, 13243 East 23rd Avenue, P.O. Box 6508, Aurora, CO 80045; e-mail: laura. brown@uchsc.edu

This work was supported by the National Institutes of Health grant 5 RO1 HD34837-06, Fetal Velocimetry and Amino Acid Transport in Pregnancy, The General Clinical Research Centers Program, National Centers for Research Resources, NIH, grant number MO1 RR00069, Research Institute of The Children's Hospital of Denver. hols are present in breast milk, adding further support of their potential importance in neonatal nutrition (8).

Although most neonatal feeding studies have focused primarily on changes in glucose and insulin concentrations $(9,10)$, there have been no studies of the impact of milk feedings upon the changes in plasma concentrations of other carbohydrates or sugar alcohols. Such studies would be important to determine the roles of these unique carbohydrates in neonatal metabolism and growth and might provide insight into their utilization within the intestine versus the liver and other organs in the body and how such patterns of utilization might reflect underlying abnormal metabolic functions. For example, although galactose is cleared by hepatic first pass metabolism during milk feeding in normal newborns $(7,11)$, this pattern might be quite different in infants with severe IUGR. It has been suspected that fetal hepatic injury might occur in severely IUGR pregnancies because of marked reduction in fetal hepatic blood flow from a reduction in umbilical blood flow and increased shunting of umbilical blood flow away from the fetal liver through the ductus venosus (DV) $(12,13)$. Postnatally, some IUGR infants demonstrate evidence of hepatic injury that might be due to such perfusion changes, especially when they are exposed to total parenteral nutrition (14-16). Even in the healthy term infant, studies have shown that the DV closes over the first several postnatal days $(17,18)$, which might affect neonatal liver function $(19,20)$. Therefore, it is reasonable to speculate that postprandial profiles of sugars normally taken up by the liver, such as galactose, might reflect hepatic injury from decreased blood flow in utero or a persistent DV shunt.

For these reasons, we measured postprandial changes in carbohydrate concentrations, including both carbohydrates and sugar alcohols, in the plasma of normal neonates following a milk feeding. We examined whether clinical variables, such as gestational age, postnatal age (PNA), milk type, feeding volume, and feeding duration, would affect the postprandial rise in plasma concentrations of the carbohydrates studied. Our goal was to establish normative postprandial data in term, healthy newborns for these carbohydrates as a basis

\footnotetext{
Abbreviations: DV, ductus venosus; [Gal], plasma galactose concentrations; [Glu], plasma glucose concentrations; IDMs, infants of a diabetic mothers; PNA, postnatal age
} 
for future study in infants with pathologic growth disturbances such as IDMs, infants with IUGR, and infants born extremely preterm. We also examined maturational changes in the postprandial plasma concentration profile of galactose over the first few weeks of postnatal life.

\section{METHODS}

Subjects. This study was approved by the Institutional Review Board at the University of Parma, Parma, Italy, and at the University of Colorado Denver, Denver, CO. Informed consent was obtained from parent or guardian of the infants before the study by Clinical Research Center nurses or study investigators. Subjects were recruited from either the level I nursery or from the NICU. Infants were considered eligible for the study if they were greater than 36 wk gestation and taking full, intermittent enteral feedings of formula or breast milk by mouth. Those infants who were initially admitted to the NICU were considered for enrollment only after any acute medical problems had resolved and the infant was at the point of receiving well baby care. For each infant studied, the following information was collected: GA, PNA, birth weight, infant weight at the time of the study, milk type, intake volume per kilogram body weight, and duration of feeding. For breast fed infants, intake volume was determined by weighing the infant before and after the feeding. Attempts were made to select infants that were taking on average $10 \mathrm{~mL} / \mathrm{kg}$ / feed over a 20-30 min time period to ensure adequate absorption of nutrients in milk from the intestine. Blood samples were obtained either by heel stick or by venous collection at baseline (immediately before the start of a feeding) and at 2-3 subsequent time points. A misinterpretation of study protocol resulted in a minor difference in sample collection between centers. Samples were correctly obtained from Denver infants at 30,60, and 90 min from baseline (start of the feeding) and from Parma infants at 30 and $60 \mathrm{~min}$ postfeed completion (range of 40-110 min postbaseline). One Parma infant also had a sample taken at $90 \mathrm{~min}$ postfeed completion (115 min postbaseline). The samples were centrifuged immediately and the plasma stored at -70 to $-80^{\circ} \mathrm{C}$ until the time of analysis.

Plasma carbohydrate analysis. Plasma was analyzed for glucose, galactose, mannose, fucose, inositol, glycerol, mannitol, erythritol, sorbitol, ribitol, glucosamine, and galactosamine as previously described (2). All plasma samples were analyzed at the University of Colorado. Briefly, the plasma was thawed quickly; $0.1 \mathrm{~mL}$ of $0.3 \mathrm{~N}$ zinc sulfate containing $30 \mathrm{mg} \%$ xylitol as an internal standard was added to $0.1 \mathrm{~mL}$ plasma, mixed well and another $0.1 \mathrm{~mL}$ of $0.3 \mathrm{~N}$ barium hydroxide was added. The mixture was centrifuged at $14,000 \mathrm{~g}$ for $10 \mathrm{~min}$ and the supernatant was filtered through a $0.45 \mu \mathrm{m}$ filter before loading on a refrigerated autosampler for HPLC analysis. A Dionex HPLC analyzer equipped with a CarboPac MA1 anion-exchange column was used for the separation of the hexoses and sugar alcohols (Dionex, Sunnyvale, CA). The analysis was run isocratically with $500 \mathrm{mM}$ sodium hydroxide for $25 \mathrm{~min}$, followed by a step change to $400 \mathrm{mM}$ sodium hydroxide (prepared with degassed, deionized water) for $20 \mathrm{~min}$ at ambient temperature. The flow rate was $0.4 \mathrm{~mL}$ per hour. A CarboPac PA 10 column was used to analyze galactose, galactosamine, and glucosamine. The system was run isocratically with $18 \mathrm{mM}$ sodium hydroxide at ambient temperature. The flow rate was 0.6 $\mathrm{mL}$ per hour. All the peaks were quantified by using a pulse amperometric detector (Dionex ED40 Electrochemical Detector) with a gold electrode. The Dionex PeakNet software was used for instrument operation and data analysis. All concentrations are expressed as micromolar.

Statistical analysis. Results are presented as median (range) unless otherwise specified. All analyses assumed a two-sided test of hypothesis with an overall significance level of 0.05 . Because of small sample sizes, nonparametric tests were used to compare baseline characteristics across centers. In addition, Fisher's exact test was used to analyze milk type across centers. Plasma galactose concentration [Gal] rise minus plasma glucose concentration [Glu] rise to second postprandial blood sample was tested using a Wilcoxon signed-rank test because of nonnormality. Longitudinal plasma carbohydrate and sugar alcohol concentrations were modeled using the mixed procedure in SAS (SAS Institute, Inc., Cary NC). A random intercept was included in all models and a random slope was considered based on a likelihood ratio test. Under the full model, a likelihood ratio test was used to determine the covariance structure (unstructured or variance components). A $\log _{\mathrm{e}}$ transformation was used to normalize [Gal]. In all analyses, milk type (breast milk, formula or both) was considered a categorical variable.

\section{RESULTS}

Twenty-six infants were evaluated $(n=9$ from Denver and $n=17$ from Parma) and all available clinical information is
Table 1. Patient clinical data by site

\begin{tabular}{lccc}
\hline & $\begin{array}{c}\text { Denver } \\
(n=9)\end{array}$ & $\begin{array}{c}\text { Parma } \\
(n=15)^{*}\end{array}$ & $p^{\dagger}$ \\
\hline Gestational age (wks) & $38(37,41.5)$ & $38(36,41) \ddagger$ & 0.25 \\
Birthweight (kg) & $2.8(2.3,3.5)$ & $3.1(2.5,4.5) \ddagger$ & 0.40 \\
Study weight (kg) & $2.8(2.4,3.5)$ & $3.2(2.5,4.3)$ & 0.14 \\
Intake volume (mL/kg) & $16.2(10.9,26.3)$ & $19.2(6.9,34.2)$ & 0.38 \\
Feed time (min) & $25(14,34)$ & $18(10,50)$ & 0.13 \\
Postnatal age (d) & $2(2,6)$ & $7(2,16)$ & 0.0008 \\
Milk type & & & $0.39 \S$ \\
$\quad$ Breast milk (BM) & $1(11 \%)$ & $5(33.3 \%)$ & \\
Formula (F) & $3(33 \%)$ & $2(13.3 \%)$ & \\
BM + F & $5(56 \%)$ & $8(53.3 \%)$ & \\
\hline
\end{tabular}

The results are given as median (range) except for milk type, which is given as $n(\%)$.

* Total $n=17,15$ patients had all available clinical data.

$\dagger$ Wilcoxon rank-sum.

$\ddagger n=16$.

$\S$ Fisher's exact.

presented in Table 1. The median PNA for the entire population of infants was $6 \mathrm{~d}(2-16 \mathrm{~d})$ and the gestational age was 38 wk (36-41.5 wk). The median volume of milk taken at each feeding (breast, formula or both) was $16 \mathrm{~mL} \cdot \mathrm{kg}^{-1}(7-34$ $\left.\mathrm{mL} \cdot \mathrm{kg}^{-1}\right)$ and the duration of feeding was $20 \mathrm{~min}(10-50$ min), which indicates that these infants were taking adequate volumes of milk over a relatively brief time period. There were no significant differences in clinical characteristics between centers, with the exception that Denver infants were studied at a significantly younger PNA (median 2 d) than Parma infants (median $7 \mathrm{~d}, p=0.0008$ ).

Figure 1 illustrates the change in [Glu] and [Gal] for each patient from baseline to the second postprandial sample, or 60-110 min after the start of the milk feeding. Despite delivery in equimolar amounts from the breakdown of lactose in milk, the median of [Glu] rise minus [Gal] rise from baseline to the second postprandial plasma sample was 674 $\mu \mathrm{M}(-38,3333 \mu \mathrm{M} ; p<0.0001)$. This observation reflects a postprandial rise in [Glu] that was 60 -fold higher than [Gal]. Clinical variables such as volume of milk ingested, gestational age, PNA, duration of feeding or milk type, or site of recruitment did not predict the change from baseline to peak concentration for either glucose or galactose.

A mixed model of [Gal] as a function of $\log _{\mathrm{e}}$ (minutes +1 ) and age revealed a significant age effect such that for each day PNA there was an $18 \%$ decrease in [Gal], holding time constant $(p=0.03)$. To further analyze the maturational age effect, infants were split into two groups, $=2 \mathrm{~d}$ PNA and $>2$ d PNA for exploratory analysis of [Gal] and [Glu] modeled separately as a function of age group and an interaction of $\log _{\mathrm{e}}$ (minutes +1) and age group through 90 min postbaseline (Table 2). [Gal] was significantly higher at all time points in the younger infants. [Glu] was also significantly higher at baseline and estimated 30 min time point, but to a lesser degree than the [Gal] differences observed. The graphs in Figure 2 display the estimated mean [Gal] with both individual term infant [Gal] and shaded 95\% confidence intervals, as well as a graphical description of the PNA effect on [Gal].

The concentrations of several other sugar alcohols and carbohydrates were measured at all time points, and baseline 


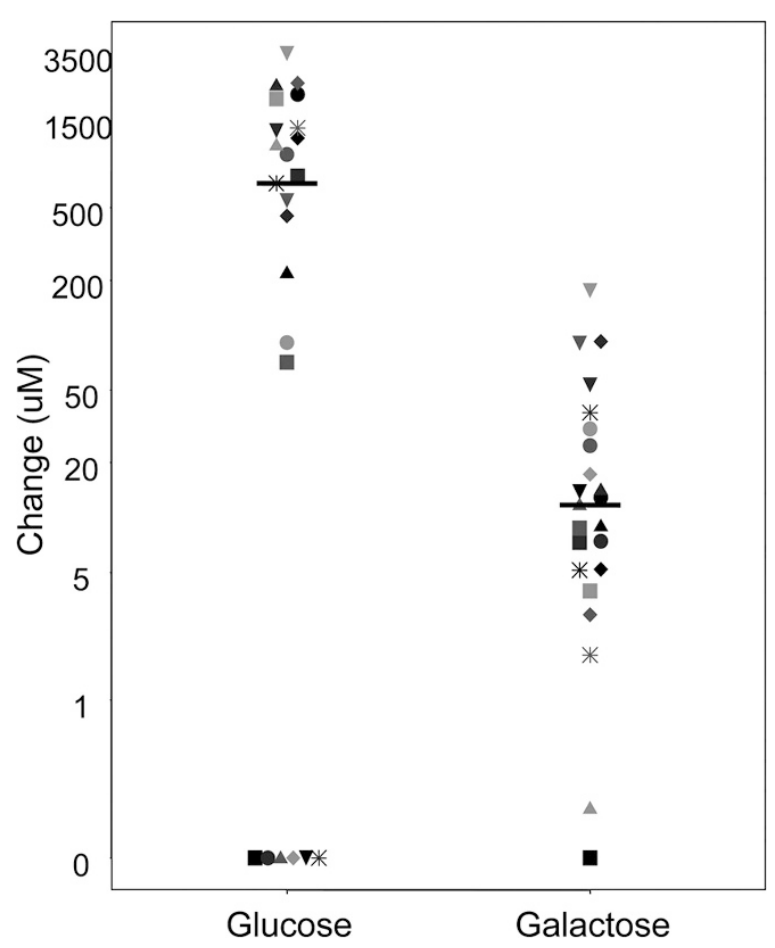

Figure 1. Change in [Glu] and [Gal] from baseline to second postprandial plasma sample. Bold horizontal bars show median change for each carbohydrate. Data points with same symbol and shade of gray represent the same subject. For the six patients without a change in [Glu], three patients demonstrated a peak in [Glu] at the first postprandial plasma sample and three patients did not demonstrate a rise in [Glu]. One of the three infants without a [Glu] peak did not have clinical data available on duration of feed or volume of intake. The remaining two patients did not demonstrate significant differences in median duration of feed time or volume of intake compared with the 17 infants with a $[\mathrm{Glu}]$ peak ( $p=0.60$ and 0.79 , respectively).

concentrations were as follows: glycerol (149.5, 40.1-357.2 $\mu \mathrm{M})$, inositol $(141.3,57.4-334.9 \mu \mathrm{M})$, mannose $(50.3,4.0-$ $110.9 \mu \mathrm{M})$, mannitol $(9.9,1.1-32.5 \mu \mathrm{M})$, and erythritol (7.5, 1.2-28.5 $\mu \mathrm{M})$. Sorbitol, ribitol, fucose, glucosamine, and galactosamine were detectable in the plasma but the concentrations were below the limits of accuracy for the HPLC methodology used (less than $5 \mu \mathrm{M}$ ). None of these hexoses and sugar alcohols showed evidence of a postprandial increase in plasma concentration. Given the lack of postprandial rise, the association between clinical variables related to the feeding and sugar alcohol concentrations could not be examined.

\section{DISCUSSION}

The present study provides normative data for the plasma concentrations of several different carbohydrates after milk feeding in a reasonable number of newborn infants. These novel results should facilitate the interpretation of feeding studies for infants with disorders of carbohydrate metabolism such as IDMs or infants with IUGR. The results also provide novel evidence of a striking difference in [Gal] between relatively younger and older neonates. Such unique developmental patterns of changes in neonatal [Gal] indicate that marked changes in hepatic blood flow and/or functional capacity might affect hepatically cleared substrates in the immediate postnatal period.

Our study demonstrates a major difference in the hepatic uptake of galactose compared with glucose. Glucose and galactose are hydrolyzed in the intestine from lactose, the principle sugar in almost all mammalian milk. Previous studies in catheterized neonatal lambs have shown that after intestinal absorption, glucose and galactose are delivered to the liver via the portal vein in equimolar amounts $(21,22)$. In this animal model, [Gal] increased significantly in the portal vein without any change in hepatic venous or femoral arterial circulation after nursing, demonstrating efficient hepatic galactose extraction in the first week of life. [Glu] increased in both hepatic and systemic circulations with no net hepatic glucose uptake. Furthermore, hepatic glucose release was observed in the fasted state (22). [Gal] measured in our study rose to only $1-2 \%$ of the [Glu] by the second postprandial plasma sample, which is consistent with previous work that galactose is almost cleared completely by the neonatal liver. We and others hypothesize that galactose derived from lactose in milk is used for the hepatic formation of glycogen and subsequent hepatic glucose release into the circulation during times of fasting $(7,22)$.

Sparks et al. in 1982 and Siegel et al. in 1988 were the first to report postprandial [Gal] in term infants $(11,23)$. In both studies, [Gal] increased significantly after a milk feeding and were within the range of 45 and $235 \mu \mathrm{M}$. The present study was in significant agreement with those studies. [Gal] were all less than $230 \mu \mathrm{M}$, though the majority of patients had [Gal] less than $55 \mu \mathrm{M}$. The present study is the first, however, to show a maturational effect on [Gal], i.e., [Gal] were higher in those infants fed in the first few days of life. Sparks et al. reported no effect of PNA on [Gal], though comparisons were made between relatively older neonates of $2,2-5$, and $5 \mathrm{wk}$ of age compared with our studies in newborn infants who were all $\leq 16 \mathrm{~d}$ of age. One mechanism that would account for this observation is a patent DV, which shunts blood from the left portal vein directly to the confluence of the hepatic veins and inferior vena cava. Studies using serial postnatal liver ultra-

Table 2. $=2 d v s>2 d$ group analysis

\begin{tabular}{lcccc}
\hline \multicolumn{1}{c}{ Group } & Baseline & $30 \min$ & $60 \min$ & $90 \mathrm{~min}$ \\
\hline Galactose $(\mu \mathrm{M})=2 \mathrm{~d}$ & $5.41(1.95,15.03)$ & $30.11(12.05,75.20)$ & $42.23(16.68,106.94)$ & $51.58(20.10,132.38)$ \\
Galactose $(\mu \mathrm{M})>2 \mathrm{~d}$ & $1.03(0.49,2.17)$ & $4.37(2.22,8.63)$ & $5.82(2.91,11.64)$ & $6.88(3.40,13.95)$ \\
$p$ & 0.01 & 0.001 & 0.001 & 0.001 \\
Glucose $(\mu \mathrm{M})=2 \mathrm{~d}$ & $4604(3874,5335)$ & $4959(4413,5504)$ & $5028(4454,5603)$ & $5070(4468,5671)$ \\
Glucose $(\mu \mathrm{M})>2 \mathrm{~d}$ & $3326(2793,3859)$ & $4252(3850,4654)$ & $4435(4008,4861)$ & $4543(4094,4991)$ \\
$p$ & 0.007 & 0.04 & 0.1 & 0.16 \\
\hline
\end{tabular}

The results are given as estimated mean with $95 \%$ confidence intervals.

$p$ value for comparison of $=2 \mathrm{~d}$ and $>2 \mathrm{~d}$ infants at each time point. 
A

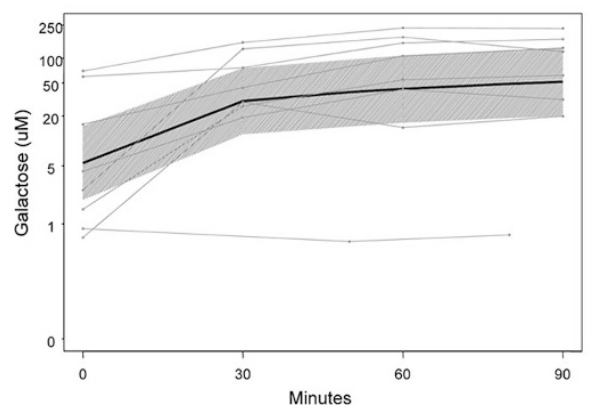

B

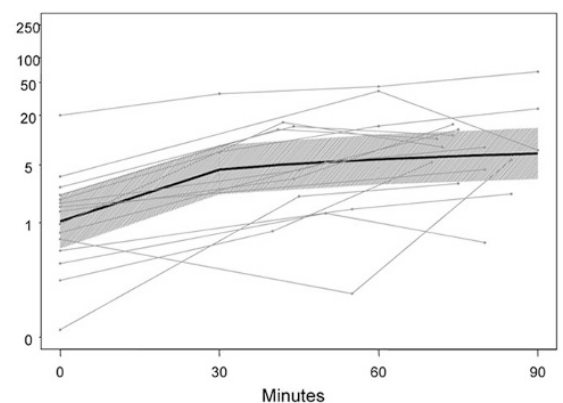

C

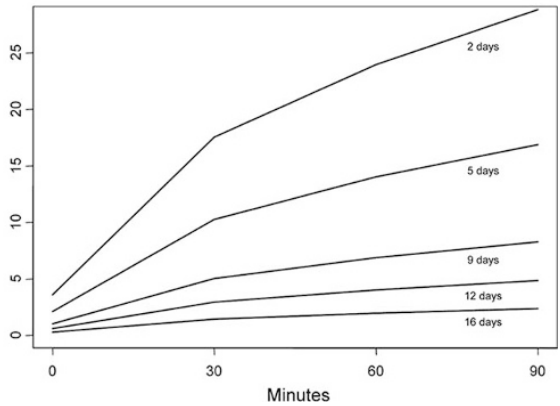

Figure 2. Estimated mean [Gal] (bold line) with $95 \%$ confidence intervals (shaded area) and subject specific postprandial data (gray lines) in (A) infants $2 \mathrm{~d}$ of age; $(B)$ infants $>2 \mathrm{~d}$ of age; $(C)$ estimated mean [Gal] over time for PNA 2, 5, 9, 12, and $16 \mathrm{~d}$.

sounds have documented that the DV may remain patent for several days after birth in term infants and for longer periods of time in preterm infants $(17,24)$. Loberant et al. (18) found that flow velocities through a patent DV were in the range of $0.15-0.70 \mathrm{~m} / \mathrm{s}$ and decreased significantly in subsequent examinations over the first 2 wk of life. Newborn lambs in the first week of life can shunt as much as $25 \%$ of blood away from the portal circulation to the systemic circulation through the DV $(25,26)$, further indicating its hemodynamic significance. Studies before ours have demonstrated a relationship between [Gal] and a patent DV in newborns. A persistent patent DV has been implicated in transient hypergalactosemia detected by neonatal mass screening for inborn errors of metabolism (20). Murayama et al. (19) found that the magnitude of blood flow through a patent DV correlated positively with [Gal] in $3 \mathrm{~d}$ old infants. Our study compared infants at several different PNAs over the first 2 wk of life and found that for each postnatal day, [Gal] fell by $18 \%$. Given the above studies that demonstrate a hemodynamically significant postnatal DV and a correlation between DV patency and galactose measurements, the findings in our study may reflect a limitation of hepatic galactose clearance because of a persistent or slowly closing DV shunt.

It also is possible that the PNA effect on [Gal] found in the present study could reflect a relative immaturity of the liver to extract galactose in the first several days of life. This option appears less likely, given that our population of infants ranged from 36 to 41 wk gestation and gestational age was nonsignificant in our longitudinal [Gal] model. Furthermore, a patent DV during the immediate 4 or 5 postnatal days has already been shown by others (17-19). This finding of a DV shunt bypassing the portal vein mandates that systemic [Gal] concentrations must rise during those postnatal days. However, further maturation of hepatic enzymatic function also may occur postnatally once the neonate begins enteral feedings regardless of gestational age. Therefore, further studies are needed to establish the relationship between DV patency and [Gal] concentrations.

In the present study, we recruited patients from two different birth centers. Since there were some minor procedural differences between sites of enrollment such as timing and method of blood draw, we analyzed whether our results differed because of site of patient enrollment. [Gal] differed between centers but only as it reflected differences in PNA.
Most of the $2 \mathrm{~d}$ old patients were enrolled in Denver and most of the patients greater than $2 \mathrm{~d}$ of age were enrolled in Parma, primarily because of differences in length of hospitalization for normal newborns in the different countries. As discussed above, this difference is most likely because of the PNA effect on [Gal] though we cannot completely exclude the effect of other unrecognized variables.

We measured the change in neonatal plasma concentrations of several other carbohydrates and sugar alcohols after a milk feeding. Cavalli et al. (8) measured free sugar and sugar alcohol concentrations in human breast milk and found that they are present but 100-fold lower than for lactose. Although several sugar alcohols, including inositol, glycerol, mannitol, and erythritol, are present in the plasma of term infants, we did not observe a postprandial increase in their plasma concentrations, probably because their concentrations in milk are relatively low, particularly compared with lactose. The concentration of oligosaccharides in human milk also is low compared with lactose; oligosaccharides account for $1 \%(\mathrm{wt} /$ vol) of human milk versus lactose which accounts for $7 \%$ of human milk (27). Carbohydrates that compose the core of oligosaccharides, such as fucose and mannose, did not have a measurable postprandial rise, presumably reflecting their relatively low concentrations in milk. Furthermore, a growing body of evidence suggests that oligosaccharides are not hydrolyzed and absorbed from the small intestine, but instead contribute to the establishment of nonpathogenic colonic flora and inhibit binding of pathogens to the intestinal epithelial cell $(28,29)$. For both of these reasons, a postprandial rise in concentration of fucose and mannose would not be expected.

In summary, this study found that glucose and galactose increase postprandially and that several other carbohydrates contained in milk do not. Further investigations are needed to define the individual roles of these carbohydrates and sugar alcohols in neonatal metabolism, whether the newborn infant produces these carbohydrates from glucose in adequate amounts to meet biologic requirements, or whether they are required nutritionally for optimal postnatal growth. Furthermore, our study continues to address the unique patterns of galactose metabolism in the neonatal diet. We demonstrate differences in [Glu] and [Gal] consistent with previous work that galactose is almost cleared completely by the neonatal liver and provide novel evidence of a marked difference in [Gal] between relatively younger and older neonates. In ad- 
dition, to the extent that [Gal] may be a sensitive marker for hepatic blood flow and/or injury from prenatal DV dilatation and shunt in pathologic conditions such as IUGR, it is of great interest to carry out similar studies in infants in whom hepatic perfusion may be compromised.

\section{REFERENCES}

1. Jauniaux E, Hempstock J, Teng C, Battaglia FC, Burton GJ 2005 Polyol concentrations in the fluid compartments of the human conceptus during the first trimester of pregnancy: maintenance of redox potential in a low oxygen environment. J Clin Endocrinol Metab 90:1171-1175

2. Brusati V, Jozwik M, Jozwik M, Teng C, Paolini C, Marconi AM, Battaglia FC 2005 Fetal and maternal non-glucose carbohydrates and polyols concentrations in normal human pregnancies at term. Pediatr Res 58:700-704

3. Teng CC, Tjoa S, Fennessey PV, Wilkening RB, Battaglia FC 2002 Transplacental carbohydrate and sugar alcohol concentrations and their uptakes in ovine pregnancy. Exp Biol Med (Maywood) 227:189-195

4. Groenen PM, Peer PG, Wevers RA, Swinkels DW, Franke B, Mariman EC, Steegers-Theunissen RP 2003 Maternal myo-inositol, glucose, and zinc status is associated with the risk of offspring with spina bifida. Am J Obstet Gynecol 189:1713-1719

5. Hallman M, Bry K, Hoppu K, Lappi M, Pohjavuori M 1992 Inositol supplementation in premature infants with respiratory distress syndrome. N Engl J Med 326:12331239

6. Howlett A, Ohlsson A 2003 Inositol for respiratory distress syndrome in preterm infants. Cochrane Database Syst Rev CD000366

7. Kliegman RM, Sparks JW 1985 Perinatal galactose metabolism. J Pediatr 107:831841

8. Cavalli C, Teng C, Battaglia FC, Bevilacqua G 2006 Free sugar and sugar alcohol concentrations in human breast milk. J Pediatr Gastroenterol Nutr 42:215-221

9. Lucas A, Boyes S, Bloom SR, Aynsley-Green A 1981 Metabolic and endocrine responses to a milk feed in six-day-old term infants: differences between breast and cow's milk formula feeding. Acta Paediatr Scand 70:195-200

10. Zarlengo KM, Battaglia FC, Fennessey P, Hay WW Jr 1986 Relationship between glucose utilization rate and glucose concentration in preterm infants. Biol Neonate 49:181-189

11. Siegel CD, Sparks JW, Battaglia FC 1988 Patterns of serum glucose and galactose concentrations in term newborn infants after milk feeding. Biol Neonate 54:301-306

12. Bellotti M, Pennati G, de Gasperi C, Bozzo M, Battaglia FC, Ferrazzi E 2004 Simultaneous measurements of umbilical venous, fetal hepatic, and ductus venosus blood flow in growth-restricted human fetuses. Am J Obstet Gynecol 190:13471358

13. Kiserud T, Kessler J, Ebbing C, Rasmussen S 2006 Ductus venosus shunting in growth-restricted fetuses and the effect of umbilical circulatory compromise. Ultrasound Obstet Gynecol 28:143-149
14. Zambrano E, el Hennawy M, Ehrenkranz RA, Zelterman D, Reyes-Mugica M 2004 Total parenteral nutrition induced liver pathology: an autopsy series of 24 newborn cases. Pediatr Dev Pathol 7:425-432

15. Baserga MC, Sola A 2004 Intrauterine growth restriction impacts tolerance to total parenteral nutrition in extremely low birth weight infants. J Perinatol 24:476-481

16. Boehm G, Muller DM, Teichmann B, Krumbiegel P 1990 Influence of intrauterine growth retardation on parameters of liver function in low birth weight infants. Eur J Pediatr 149:396-398

17. Kondo M, Itoh S, Kunikata T, Kusaka T, Ozaki T, Isobe K, Onishi S 2001 Time of closure of ductus venosus in term and preterm neonates. Arch Dis Child Fetal Neonatal Ed 85:F57-F59

18. Loberant N, Barak M, Gaitini D, Herskovits M, Ben Elisha M, Roguin N 1992 Closure of the ductus venosus in neonates: findings on real-time gray-scale, colorflow Doppler, and duplex Doppler sonography. AJR Am J Roentgenol 159:10831085

19. Murayama K, Nagasaka H, Tate K, Ohsone Y, Kanazawa M, Kobayashi K, Kohno Y, Takayanagi M 2006 Significant correlations between the flow volume of patent ductus venosus and early neonatal liver function: possible involvement of patent ductus venosus in postnatal liver function. Arch Dis Child Fetal Neonatal Ed 91:F175-F179

20. Nishimura Y, Tajima G, Dwi BA, Sakamoto A, Ono H, Sakura N, Naito K, Hamakawa M, Yoshii C, Kubota M, Kobayashi K, Saheki T 2004 Differential diagnosis of neonatal mild hypergalactosaemia detected by mass screening: clinical significance of portal vein imaging. J Inherit Metab Dis 27:11-18

21. Kaempf JW, Li HQ, Groothuis JR, Battaglia FC, Zerbe GO, Sparks JW 1988 Galactose, glucose, and lactate concentrations in the portal venous and arterial circulations of newborn lambs after nursing. Pediatr Res 23:598-602

22. Spedale SB, Battaglia FC, Sparks JW 1992 Hepatic metabolism of glucose, galactose, and lactate after milk feeding in newborn lambs. Am J Physiol 262:E46-E51

23. Sparks JW, Avery GB, Fletcher AB, Simmons MA, Glinsmann WH 1982 Parenteral galactose therapy in the glucose-intolerant premature infant. J Pediatr 100:255-259

24. Loberant N, Herskovits M, Barak M, Ben Elisha M, Herschkowitz S, Sela S, Roguin N 1999 Closure of the ductus venosus in premature infants: findings on real-time gray-scale, color-flow Doppler, and duplex Doppler sonography. AJR Am J Roentgenol 172:227-229

25. Botti JJ, Edelstone DI, Caritis SN, Mueller-Heubach E 1982 Portal venous blood flow distribution to liver and ductus venosus in newborn lambs. Am J Obstet Gynecol 144:303-308

26. Townsend SF, Rudolph CD, Rudolph AM 1989 Changes in ovine hepatic circulation and oxygen consumption at birth. Pediatr Res 25:300-304

27. Stahl B, Thurl S, Zeng J, Karas M, Hillenkamp F, Steup M, Sawatzki G 1994 Oligosaccharides from human milk as revealed by matrix-assisted laser desorption/ ionization mass spectrometry. Anal Biochem 223:218-226

28. Engfer MB, Stahl B, Finke B, Sawatzki G, Daniel H 2000 Human milk oligosaccharides are resistant to enzymatic hydrolysis in the upper gastrointestinal tract. Am J Clin Nutr 71:1589-1596

29. Coppa GV, Pierani P, Zampini L, Bruni S, Carloni I, Gabrielli O 2001 Characterization of oligosaccharides in milk and feces of breast-fed infants by highperformance anion-exchange chromatography. Adv Exp Med Biol 501:307-314 